\title{
EPIDEMIOLOGY OF FILARIASIS
}

\section{EXPOSURE AND THE DYNAMICS OF LYM- PHATIC FILARIASIS INFECTION}

\author{
SRIVIDYA A.* DAS P.K.*, RAMAIAH K.D.*, GRENFELL B.T.**, \\ MICHAEL E*** \& BUNDY D.A.P.***
}

KEY WORDS : Iymphatic filariasis, epidemiology. vector biology.

central task in the epidemiology of lymphatic filariasis is

to quantify the dynamics of parasite transmission from the vector to the human population and vice versa. In particular, there is a need to quantify the relationship between infection dynamics in the vector population and the age patterns of human infection and disease. This not only forms the first step in the building of an overall dynamic transmission model for lymphatic filariasis but, in addition, is also crucial in the context of modelling the effects of vector control interventions. Linking transmission dynamics with the age-incidence of infection is also essential for examining the potential role of immunity in regulating infection and disease in endemic communities. Here, we use parallel, longitudinal entomological and parasitological data collected during and after a 5-year vector control programme in Pondicherry, South India, to address the problem of how changes through time in the density of biting mosquitoes affect the acquirance of Wuchecheria bancrofti infection in young children, as measured by microfilarial ( $\mathrm{mf}$ ) age-prevalence. The data were collected in the period 1981 to 1989 , and allow us to quantify the effects of an Integrated Vector Management (IVM) programme, which reduced the density of the main vector (Culex quinquefasciatus) by as much as $80 \%$ during the period 1981 to 1985 (Subramanian et al., 1989 ; Das et al., 1992) (Fig. 1a). Note that the index of infection from the vector population is measured by ITv, which is defined as the intensity of transmission with respect to vector population, and denotes the actual number of L3 larvae inoculated into a person per hour of exposure to parous biting mosquitoes (see details of calculation in Ramaiah \& Das, 1992). Entomological data were collected yearly from resting stations from 1981 to 1989, but the corresponding human parasitological data for this study were collected from the IVM area via mass blood surveys (using $20 \mu \mathrm{l}$ blood samples) in 1981 (pre-control), and one and four years post-control in 1986 and 1989 respectively (for full details of survey design see Subramanian et al., 1989 ; Das et al., 1992). Vector control significantly reduced the prevalence of infection in children age $\leq 10$ years ( $p<0.001$ ) (Fig. 1b), however, the longevity of adult parasites lessened the difference between pre-control (1981) and post-control $(1986,1989)$ infection rates in adults (data not shown). The following analysis therefore concentrates on $\mathrm{mf}$ age-prevalence in children $\leq 10$ years. The age-prevalence curves in Fig. 1b indicate that the impact of vector control

\footnotetext{
* Vector Control Research Centre, Medical Complex, Indira Nagar, Pondicherry - 605006 India.

** Department of Zoology, University of Cambridge, Downing Street, Cambridge CB2 3EJ, UK.

*** Parasite Epidemiology Research Group, Department of Biology, Imperial College, Prince Consort Road, London SW7 2BB, U.K.
}

after 1981 not only reduces the infection rate in young children, but also apparently increases the observed pre-patent period from around 3 years in 1981 to 4 to 5 years in 1986 and 1989. The dynamics of vector control, and hence the relationship between infection dynamics in the vector and human populations, particularly children, can be modelled as follows. Assuming that few children of age $\leq 10$ years lose infection after gaining it, we can model the gain of infection in this age group by the following modification of the standard catalytic age-prevalence equation (Hairston \& Jachowski, 1968 ; Vanamail et al., 1989) :

$$
I(a)=\left(\begin{array}{ll}
0 & a \leq a_{0} \\
1-\exp \left(-\int_{a_{0}}^{a} \lambda(s) d s\right) & a>a_{0}
\end{array}\right)
$$

for $I(O)=0$. Equation (1) describes the rate of increase of the proportion infected (I), as a function of age, $a$. The parameter $\lambda$ (per age) is the per capita infection rate, or force of infection, and $a_{0}$ is the prepatent period.

We use ITv, the index of infective vector biting density, in order to convert this age prevalence curve into prevalence as a function of previous exposure to infection model. First, we define $X$, the cumulative past exposure to infection of an individual age ' $a$ ' in year ' $t$ ' in terms ITv, as follows

$$
X(a, t)=\sum_{i=1}^{a-1} \operatorname{ITV}(t-i)
$$

(for example, 3 year olds in 1985 have experienced infection in 1983 and 1984) leading to the following modification of equation (1):

$$
I(X)=\left(\begin{array}{ll}
0 & X \leq X_{0} \\
1-\exp \left(-\int_{x_{0}}^{x} \lambda^{\prime}(s) \mathrm{ds}\right) & X>X_{0}
\end{array}\right)
$$

(approximating the discrete variable $X$ as a continuous variable) $\lambda^{\prime}(X)$ (units $X^{1}$ ) is now the force of infection as a function of cumulative past exposure rather than age and $X_{0}$ measures the prepatent period in terms of previous infective biting exposure.

Fig. 2a shows the observed mf age-prevalence curves for 1-10 year olds in the years 1981, 1986 and 1989, plotted against cumulative intensity of transmission $(X)$. The change of scale from age to intensity brings the prevalences from the 3 years into similar curves - an analysis of covariance with binomial errors illustrates that the prevalence from the 3 years were not significantly different $(p=0.22$ ) over their common range of $X$. Fig $2 \mathrm{~b}$ shows the maximum likelihood fit to these data of the catalytic model defined by equation (3), and clearly supports the hypothesis that differences in the $\mathrm{mf}$ age-prevalence in young children observed before and after a control programme simply reflect changes in their cumulative past exposure to infection. In particular, the 3 age-prevalence curves all now have the same intercept on the $\mathrm{X}$ axis, indicating that a total exposure of around $\mathrm{X}_{0}=0.105$ is required to produce a patent infection. This threshold was achieved in 3 years in the pre-vector control period (1981) and 4-5 years post control (1986 and 1988). As described by Rajagopalan et 

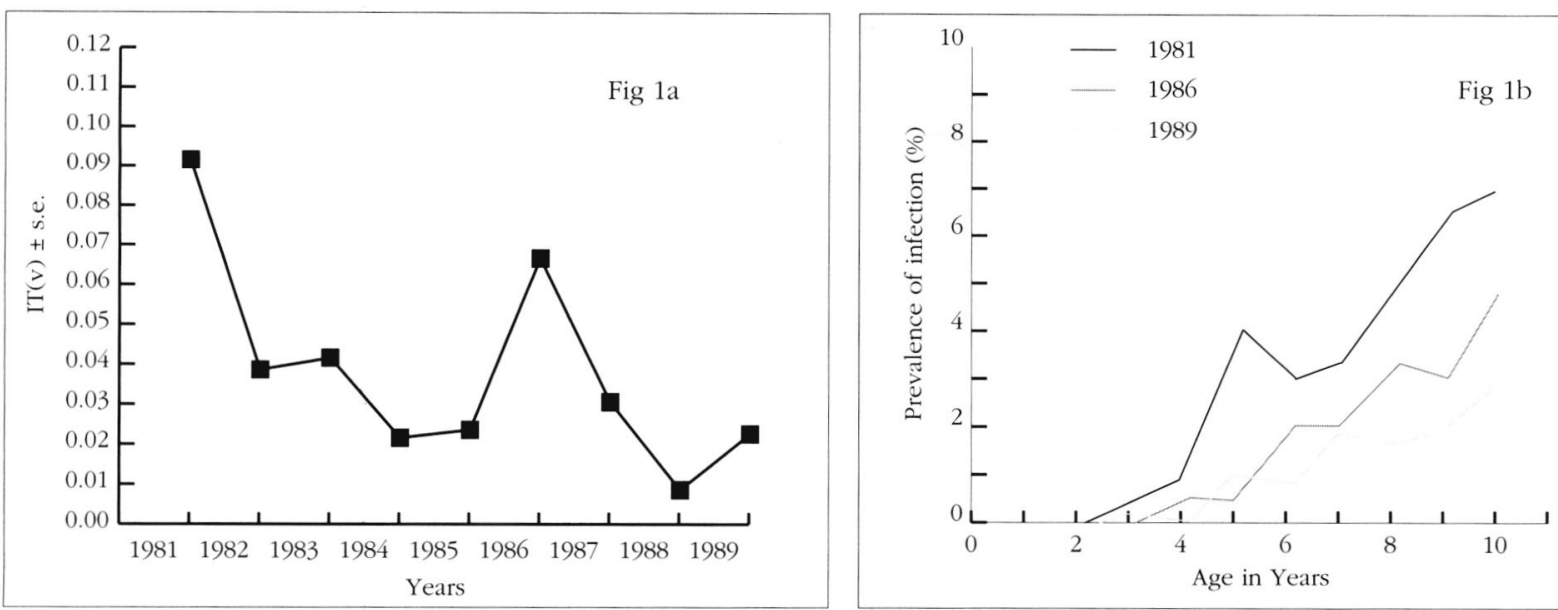

Fig. 1. - (a) Intensity of transmission in the IVM area in terms of the vector population (ITv) during the years 1981-1989. (b) Comparison of age specific mf-prevalence in young children (up to 10 years) during 1981, 1986 and 1989.
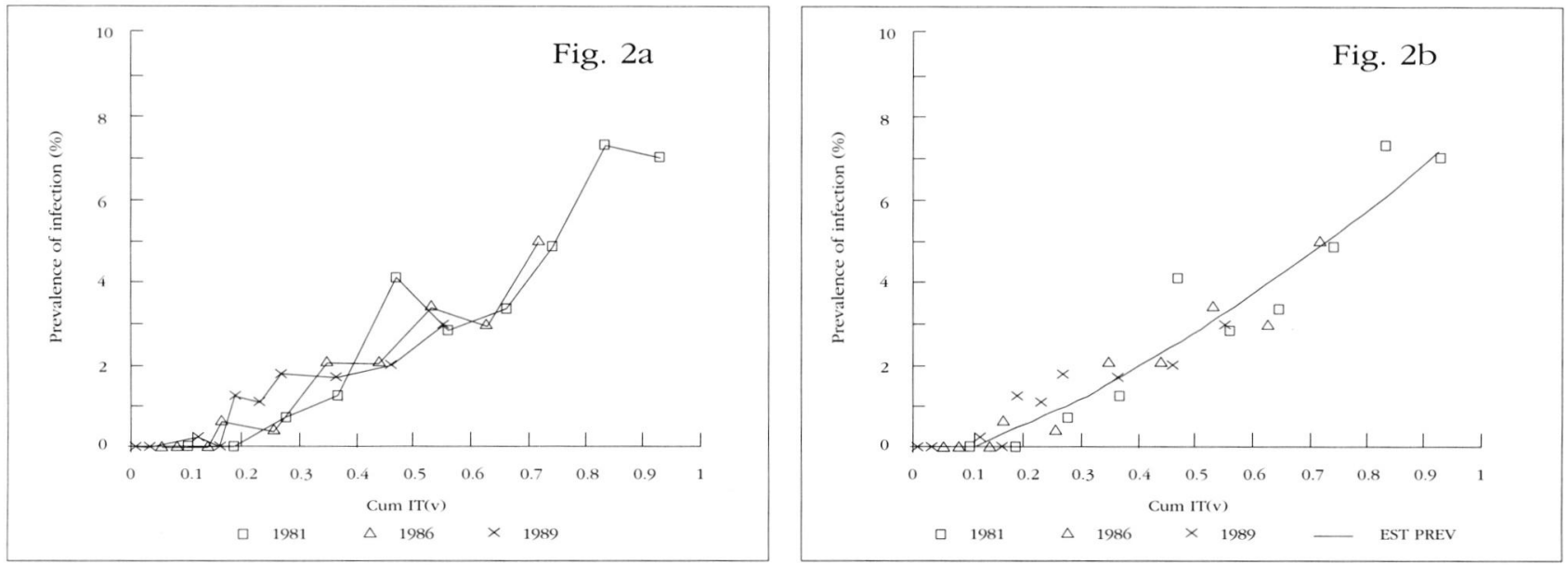

Fig. 2. - (a) Comparison of age specific mf-prevalence of children during 1981, 1986 and 1989 scaled in relation to cumulative ITv. (b) Fit of the force of infection model linking age specific mf-prevalence to cumulative ITv in children. Symbols denote the observed age specific mf prevalences in children up to 10 years in 1981, 1986 and 1989, while the solid curve represents the fit of the catalytic model defined by equation (3) in the text.

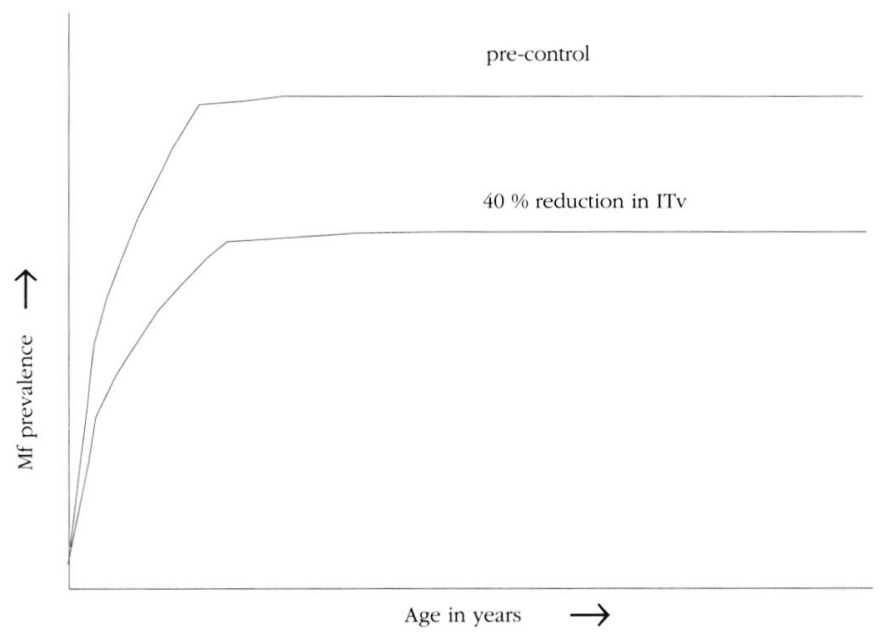

Fig. 3. - Predicted change in the age-prevalence of microfilaraemia as a result of reducing the biting vector density to $40 \%$ of the pre-control level. The standard (reversible) catalytic age prevalence model (Hairston \& Jachowski, 1968 ; Vanamail et al., 1989) was used to model the equilibrium age-prevalence $\mathrm{mf}$ curve following vector control assuming that a reduction in ITv simply decreases the force of infection $\lambda$ by the same factor. Both and $\lambda$ and $\mu$, the instantaneous rate of loss of infection, are taken to be constants and independent of age respectively. 
al. (1977), this corresponds to a total of around 3145 infective bites which is bracketed by the range of previous estimates. The model also has several implications for the performance of vector control strategies. In particular, the fact that the rise in $\mathrm{mf}$ prevalence in children depends only on the average level of transmission scaled by age, allows us to model the eventual equilibrium effects of constant levels of vector control. Fig. 3 illustrates the application of this simple model, and portrays the change expected at equilibrium to the pre-control age-prevalence curve as a result of a $40 \%$ reduction in ITv [as occurred in the IVM area over the 8-year period from 1982 to 1989 (Fig. 1)]. The result indicates that vector control alone may have little impact on the overall age-prevalence of infection even when sustained for long periods. These results are crude and, for instance, ignore spatial heterogeneities and the likely effects of acquired immunity in adults. The present work, however, provides an initial framework for assessing the impacts of these processes in the overall transmission dynamics and control of filariasis.

\section{REFERENCES}

Das P.K., Manoharan A., Subramanian S., Ramaiah K.D., Pani S.P., Rajavel A.R. \& Rajagopalan P.K. : Bancroftian filariasis in Pondicherry, South India - epidemiologic impact of recovery of the vector population. Epidemiology and Infection, 1992, 108, 483-493.

Hairston N.G. \& JACHOWSKi L.A. Analysis of the Wuchereria bancrofti population in the people of American Samoa. Bulletin of the World Health Organization, 1968, 38, 29-69.

Rajagopalan P.K., Kazmi S.J. \& Mani T.R. : Some aspects of transmission of Wuchereria bancrofti and ecology of the vector Culex pipiens fatigans in Pondicherry. Indian Journal of Medical Research, 1977, 66, 200-215.

RAMAIAH K.D. \& DAS P.K. : Seasonality of adult Culex quinquefasciatus and transmission of bancroftian filariasis in Pondicherry, South India. Acta Tropica, 1992, 50, 275-283.

Subramanian S., Pani S.P., Das P.K. \& Rajagopalan P.K. : Bancroftian filariasis in Pondicherry, South India : 2. Epidemiological evaluation of the effect of vector control. Epidemiology and Infection, 1989, 103, 693-702.

Vanamail P., Subramanian S., Das P.K., Pani S.P., Rajagopalan P.K. Bundy D.A.P. \& GRENFEll B.T. : Estimation of age-specific rates of acquisition and loss of Wuchereria bancrofti infection. Transactions of the Royal Society of Tropical Medicine and Hygiene, 1989, 83, 689-693.

\section{CATTLE, WORMS AND ZOOPROPHYLAXIS}

\section{RENZ, A.*, ENYONG P.** \& WAHL G.***}

KEY WORDS : onchocerciasis, crossprotective immunity. zooprophylaxis, epidemiology. mathematical model. Cameroon.

\footnotetext{
SUMMARY

Epidemiological studies in North Cameroon indicate that a high population density of cattle in relation to man protects from severe oncho-

*Fachgebiet Parasitologie, Universität Hohenheim, Emil-WolfStrasse 34, 70599 Stuttgart, Germany

** Medical Research Station, P.O. Box 55, Kumba, Cameroon.

*** Institut für Tropenmedizin, Wilhelmstrasse 27, 72074 Tübingen, Germany.
}

cerciasis. In the following, we present a mathematical model which allows to study the effects of zoophily of the vectors, of concomitant immunity in the human population and of cross-protective immunity caused by the infective larvae of a bovine parasite, Onchocerca ochengi, inoculated into man by Simulium damnosum s.l. Besides this influence on onchocerciasis, cattle also play a major role in the epidemiology of malaria (zooprophylaxis through vector dilution) and schistosomiasis (protection by crossreactive concomitant immunity).

\section{INTRODUCTION}

n many traditional African societies, cattle are a sign of richness and welfare. There is also increasing evidence that cattle protect from some of the most dangerous parasitic diseases. Either by diverting the vectors coming to take a bloodmeal or by the transmission of bovine parasites to man which do not develop but stimulate the immune system. The reduction of the vectorial capacity of bloodfeeding vectors has been called "zooprophylaxis", whilst the latter, i.e. resistance against the human parasite caused by cross-reactive immunity, was named the "Jennerian principle of zooprophylaxis" (Nelson, 1987). In the following, the role of cattle will be examined with view to the epidemiology of human onchocerciasis, with a brief outlook on its influence on malaria and schistosomiasis, which are usually co-endemic in the same regions.

\section{A MATHEMATICAL MODEL FOR ONCHOCERCIASIS}

A stochastic model has been derived from the formulae given in Dietz (1982). It allows to plot the average worm load in the human population (adult worms or microfilariae in the skin) in dependence from the size of the vector populations $(\mathrm{m})$ and their biting rate on man (h, proportion of bloodmeals on the human host). In Figure 1, the effect of bloodfeeding on animals (zoophily) alone is considered, whilst in Figure 2, the probability of an infective larvae of $O$. volvulus to reach maturity in the human host is reduced by $90 \%$. Such a reduction could either stem from crossprotective immunity caused by $O$. ocheng $i$ L3 or from a vaccination programme.

Even at moderate levels of cross-protection (50\% reduction) and bloodfeeding on non-human hosts (50\% of all bloodmeals) the treshold level for endemicity, below which onchocerciasis cannot maintain itself, increases from an Annual Biting rate of 200 flies per man and year (if all flies fed on man and there is no immunity) to 1500 flies per man and year. However, the protection by immunity would have to achieve or even surpass $99,9 \%$ to eradicate onchocerciasis under the worst conditions, i.e. at places as observed in the Cameroon rain forest, where the Annual Biting Rate at the river is as high as 600000 Simulium damnosum s.l. per man and year (Duke et al., 1972) and where almost all flies fed on the human population.

Whilst our model predicts that an increase in the proportion of bloodmeals and animals (cattle) is very unlikely to increase transmission of human onchocerciasis (for example by an increase of the fly-population due to better survival and/or fecundity), and this is supported by our field data, the situation may be different with regard to the transmission of malaria by Anopheles. 